\title{
Inguinal hernia repair at the Aga Khan Hospital, Nairobi: Practice and preference discordance
}

\author{
R. Wasike, MBChB, MMed (Surg) and A. Abdallah, MBChB, H Dip Surg (SA), FRCS, Department of \\ Surgery, Aga Khan University Hospital, P.O. Box 30270, 00100, Nairobi, Kenya \\ Correspondence and reprint requests to: \\ Dr. R. Wasike, Department of Surgery, Aga Khan University Hospital, Nairobi, P.O. Box 30270, 00100, \\ Nairobi, Kenya, Email: ronald.wasike@aku.edu
}

\section{Abstract}

OBJECTIVES: To review the current hernia repair methods at the Aga Khan University Hospital (AKUH) and relate to the preferred method of choice for groin hernia repair among the practicing general surgeons.

DESIGN: An audit from retrospective practices.

SETTING: A tertiary referral hospital, Aga Khan University Hospital, Nairobi.

SUBJECTS: All elective adult hernia repairs performed at the Aga Khan University Hospital, Nairobi between 2004-5 by all surgeons with admitting privileges were reviewed. The methods of repair were noted. A questionnaire was also availed to the admitting surgeons to indicate their preferred method, and this was correlated with the audit results.

RESULTS: One hundred and seventy four hernia repairs were reviewed, 58 (33.72\%) were left sided, 95 (54.07\%) were right sided and 21 $(12.21 \%)$ were bilateral. The most common method of repair was the Modified Bassini's, performed in $99(56.9 \%)$ repairs. The survey results however, indicated that three quarters of the practicing surgeons preferred non-tension repairs. The mean hospital stays were similar for the tissue and mesh repairs.

CONCLUSION: Tissue repair is still practiced and favored at our institution by the surgical fraternity. This practice is at variance with the stated preference for mesh repairs. In the absence of outcome data for this local practice, it is difficult to justify its utility in an environment where meshes are easily available.

\section{Introduction}

The repair of groin hernias is a common and important surgical procedure. In Africa, groin hernias comprise an even greater percentage of surgical volume and account for more morbidity (1) than in Western practice. The introduction of the Bassini repair in the early part of the $20^{\text {th }}$ century dropped the recurrence rates from $100 \%$ to about $10 \%$. Even better outcome was recorded for Shouldice repair which for some time remained the standard against which hernia repairs were judged. In the last 20 years however, a total revolution in surgical repairs with the introduction of tension-free (mesh) methods has been witnessed (2). There is ample evidence now showing mesh repairs to have the lowest rates of recurrences even in non-specialized centres (3). Knowledge about evidence is however, not always associated with a change in practice. Further, tissue repairs continue to flourish in environments where resources are constrained (4) or perceptions of untoward economic impact in the use of the mesh are held. 
The Aga Khan University hospital is a tertiary referral and teaching hospital in Nairobi. This study evaluated the groin hernia practice at the institution and correlated the practice with the preferred hernia repair method as stated by the surgeons.

\section{Methods}

This was a retrospective audit on the practice of groin hernia repairs over two years (20042005). Data abstracted from patient records included side of hernia, method of repair and hospital stay. A questionnaire was availed to willing admitting surgeons who had performed herniorrhaphies during this period to indicate their preferred method of groin hernia repairs. The surgeons' preferred methods were then compared with the methods abstracted from patient records. The results are presented in terms of proportions and discussed in the light of literature recommendations.

\section{Results}

A total of one hundred and seventy four hernia repairs were reviewed. It was established that ninety nine $(56.9 \%)$ of the total repairs were achieved by tissue repairs (modified Bassini's). Sixty seven repairs (38.5\%) were open mesh (Lichtenstein) and eight, making up 4.6\% were repaired laparoscopically.
The mean hospital stay for patients who underwent tissue repairs was 2.4 days (range 1-18 days). Those who underwent open (Lichtenstein) mesh repair stayed for a mean of 2.3 days (range 1-11 days). The differences in the lengths of hospital stays were statistically insignificant.

Twenty admitting surgeons (response rate $80 \%)$ responded to the survey question. All the surgeons performed less than 5 hernia repairs per month. Three quarters $(n=15,75 \%)$ of the surgeons said mesh repair was their method of choice (Figure 1)

\section{Discussion}

The present results indicate the most common practice of groin hernia repair at our institution was the modified Bassini's method (56.9\%). This was a surprising result especially considering that three-quarters of surgeons surveyed preferred a non-tissue repair. In Basini repair, like other sutured repairs, the tissues are under a certain amount of tension and this results in a break down of the repair and subsequent recurrence. The quoted rate of recurrence for the Bassini's repair is high, peaking 10\% when performed in non-specialty centers (5). A more forgiving tissue repair is the Shouldice operation, with reported recurrence rates of $2 \%$ in non-speciallty centers (5) and $0.1 \%$ in specialty clinics $(3,5)$. Ostrow (1) has argued for this method of repair in situations where,

Figure 1: Preferred method of repair as indicated by surgeons

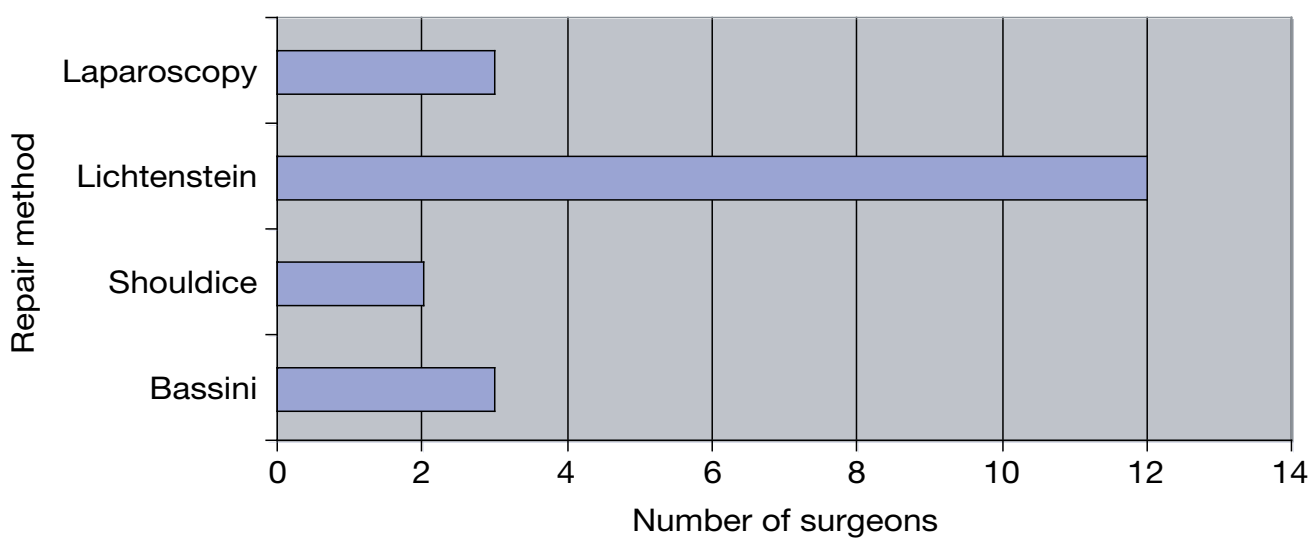


for resource reasons, meshes cannot be used. In the current study, the Shouldice method was only performed for three patients. It is likely that the difficulty involved in acquisition of the technique explained its utility in this study. We echo Ostrow's sentiments and suggest that our local universities should train surgical residents in this method of repair for groin hernias. In many parts of the country where majority of the hernias are treated, resources and mesh availability may be real concerns.

Tension-free repairs (both open and laparoscopic) were performed for $43.1 \%$ of the hernias. Lichtenstein (7) advocated an open onlay mesh repair applied on the internal oblique fascia in 1986. After this, numerous mesh systems have been developed using this principle including plug repairs, plug and onlay meshes and the prolene hernia system (8). The latter is one of several methods where the preperitoneal space (Bogros) is prepared for mesh placement. These mesh techniques are easily acquired (9), performed quickly under local anesthesia with very good results. Randomized controlled trials (RCTs) (2) have gone beyond depicting superiority of mesh repairs over even shouldice repair $(10,11)$ to now comparing the different types of mesh delivery systems. Two RCTs $(12,13)$ for example, have compared various mesh repairs without showing any significant differences in recurrence rates. The debate on open versus laparoscopic repairs is also not about recurrence but costs and morbidity related to mesh hernioplasty.

The two major laparoscopic approaches are trans-abdominal preperitoneal repair (TAPP) and total extra peritoneal repair (TEP). These procedures are costly, difficult to learn and require general anesthesia (14). Nerve irritation is a more common complication. Johannson and Bringman (15) showed that patients undergoing LHR had faster recovery times, but the procedure incurred significant increased costs. In Rattner's Multicentre RCT (16), patients undergoing LHR had more serious complications. In a meta-analysis, Memon (17) confirmed less post-operative pains, improvements in recovery times and increased costs with LHR. O'Dwyer (18) has summarized the current status of LHR versus OHR (with mesh). He stated that the drawbacks of LHR are necessity of general anesthesia, longer learning curve, increased costs and more serious complications, precluding its general use. Open hernia repair (OHR) using mesh would seem to be the procedure of choice for primary inguinal hernias, and LHR useful for for recurrent and bilateral hernias (19).

In conclusion, the modified Bassini repair is a common hernia repair method in our institution despite the preference for meshrepair, availability of meshes and absence of local outcome data to support the practice. Training and proficiency in the shouldice and open mesh techniques should form parts of core competencies in the surgical trainee programs in the country.

\section{References}

1. Ostrow B. What is the most appropriate repair for groin hernias in Africa. Surg. Afr. 2005; 65:231-239.

2. Kingsnorth A. and Leblanc K. Hernias: Inguinal and incisional. Lancet. 2003; 362: 1561-1571.

3. Shouldice E.B. The Shouldice repair for groin hernias Surg. Clin. N. Amer. 1993; 83: 1163-1187.

4. Odulato A. and Sancehz-Cabezudoc. The Shouldice herniorrhaphy in the treatment of inguinal hernias: Prospective study on 775 patients. Hernia. 2004 8: 60-63.

5. Paul A., Jroidl H., Williams J.I., Rixen D. and Langen R. Randomized trial of modified Bassini's versus Shouldice inguinal hernia repair: The Cologne Study Group. Brit. J. Surg. 1995; 82: 1531-1534.

6. Welsh Dr. and Alexander M.A. The Shouldice repair. Surg. Clin. North. Amer. 1993; 73: 471-485.

7. Kuzar M., Belsham P.A. and Karj A.E. The Lichtenstein repair for groin hernias. Surg. Clin. N. Amer. 200383 : 1099-1117.

8. Mayagoitia J.C. Inguinal hernioplasty with prolene hernia system. Hernia. 2004; 8(1): 64-66.

9. Mokete M. and Earnshaw J. J. Evolution of an inguinal hernia surgery practice. Postgrad. Med. J. 2001; 77(905): 188-190.

10. Nordin P., Bartelmess P., Janssonc, et al. Randomized trial of Litchenstein versus Shouldice repair. In general surgery practice. Brit. J. Surg. 2002; 89: 45-49. 
11. Scott N.W., McCormack K., Graham P., et al. Open mesh versus non mesh for groin hernia repair. The Cochrane Database Syst. Rev. 2005; 2CDOO2197.

12. Nienhuijs S.W., Van Oort I., Keemers-Gels M.E., et al. Randomized clinical trial comparing the prolene hernia system, mesh plug repair and Lichtenstein method for open inguinal hernia repair. Brit. J. Surg. 2005 92: 33-38.

13. Muldoon R.L., Marchant K., Johnson D.O., et al. Lichtenstein versus preperitonial prosthetic mesh placement in open inguinal repair, a prospective, randomized trial. Hernia. 2004 8: 98-103.

14. Swanstrom L.L. Laparoscopic hernia repairs. The importance of cost as an outcome measurement at the century's end. Surg. Clin. North Amer. 2000; 80(4): 1341-1351.
15. Bringman S., Ramel S., Heikkinen T.M., et al. Tension free inguinal hernia repair: TEP versus mesh plug versus Lichtenstein: A prospective randomized controlled trial. Ann. Surg. 2003; 237(1): 142-147.

16. The MRC laparoscopic groin hernia trial group. Laparoscopic versus open repair of groin hernia: A randomized comparison. Lancet. 1999 354: 185-190.

17. Memon M.A., Cooper N.J., Memon B., et al. Meta analysis of randomized clinical trials comparing open and laparoscopic inguinal hernia repair. Brit. J. Surg 2003 90: 1479-1492.

18. O'Dwyer P.J. Current status of the database on laparoscopic hernia repair. Brit. Med. Bull. 2004 70: 105-118.

19. Voyles C.R. Outcomes analysis for groin hernia repairs. Surg. Clin. North Amer. 2003 83: 1279-1287. 